\title{
A LINGUAGEM AUDIOVISUAL NA PRODUÇÃO PARTILHADA DO CONHECIMENTO NA COMUNIDADE XAVANTE DE SANGRADOURO-MT
}

\section{AUDIOVISUAL LANGUAGE IN SHARED KNOWLEDGE PRODUCTION IN THE XAVANTE COMMUNITY - SANGRADOURO-MT}

Jonathas Beck Ramos ${ }^{1}$

Resumo: O presente artigo apresenta reflexões conceituais, memórias e narrativas sobre as vivências entre pesquisadores da Universidade de São Paulo, indígenas Xavantes e Bororos na aldeia de Sangradouro - MT. A partir de uma oficina de audiovisual promovida pelo Ceddip (Centro de estudos de pesquisa digital partilhada - Diversitas USP) foram criados processos de troca de conhecimento e comunhão de autorias em dinâmicas de produção de vídeos com temáticas deliberadas por rodas de conversa. Chamamos aqui a proposta de produção de conhecimento entre universidade e comunidade de Produção Partilhada do Conhecimento. Subvertendo a lógica tradicional de pesquisa dicotômica entre objeto de pesquisa e pesquisador, a proposição de Produção Partilhada do Conhecimento tem como objetivo favorecer a autoria e a participação ativa da comunidade não acadêmica na pesquisa. Na ocasião, grupos indígenas Xavantes e estudantes da Universidade de São Paulo puderam juntos criar vídeos sobre a cultura Xavante.

Palavras-chave: Linguagem Audiovisual. Produção Partilhada do Conhecimento e Cultura Oral. Cultura digital.

Abstract: This article presents conceptual reflections, memories and narratives about the experiences among researchers from the University of São Paulo, indigenous Xavantes and Bororos in the village of Sangradouro - MT. From an audiovisual workshop promoted by Ceddip (Center for Shared Digital Research Studies Diversitas USP), processes for the exchange of knowledge and the sharing of authorship were created in the dynamics of video production with themes deliberated by conversation circles. We call here the proposal for the production of knowledge between the university and the community of Shared Production of Knowledge, subverting the traditional logic of dichotomous research between research object and researcher, the proposition of Shared Production of Knowledge aims to favor authorship and the active participation of the non-academic community in research. On the occasion, Xavante indigenous groups and students from the University of São Paulo were able to create videos about the Xavante culture.

Key words: Audiovisual language, Shared Production of Knowledge, Oral Culture and digital culture.

Data de submissão: 18.04 .2021

Data de aprovação: 08.09.2021

Identificação e disponibilidade:

(https://revista.univap.br/index.php/revistaunivap/article/view/2589,

\footnotetext{
1 Doutorando em linguagem hipermídia pelo programa interdisciplinar DIVERSITAS -USP, E-mail: jonathas.beck@gmail.com.
} 
http://dx.doi.org/10.18066/revistaunivap.v27i54.2589).

\title{
1 INTRODUÇÃO
}

O presente artigo aborda aspectos da Produção Partilhada do Conhecimento, proposição em que a construção do conhecimento é realizada de modo partilhado a partir de metodologias que buscam aproximar saberes diversos e que incluam diferentes matrizes de linguagem na produção de conhecimento científico, como imagem e som. A partir desta premissa pensamos a possibilidade de produzirmos conhecimento não mais sobre comunidades e não apenas pelo texto escrito, mas com interlocutores destas comunidades a partir da escuta sensível e da autorepresentação na linguagem audiovisual. Como aponta Lazaneo (2017), expondo sobre a produção partilhada do conhecimento:

\begin{abstract}
Poderíamos de algum modo refletir que, em Produção Partilhada, o pesquisador também é um "nativo" para o "nativo", e o habitualmente considerado "nativo" é também ele um "pesquisador". [...] Esta premissa porém, não fundamenta-se nem sob uma ideia de homogeneização do conhecimento (supostamente entre o pesquisador e o nativo, um binômio insuficiente como vimos) e nem, tampouco, de exclusão da diferença mas, de outro modo, da conjugação dos sentidos, afetos e diferentes formas possíveis de formação do conhecimento inauguradas pelo encontro. (LAZANEO, 2017, p. 117)
\end{abstract}

Consequência da premissa segregacionista e racionalista foi a marginalização de culturas de tradição oral no contexto da produção de conhecimento científico em nível institucional, pois a valorização da palavra escrita enquanto "ritual" exclusivo das instituições acadêmicas, consequência da lógica racionalizante do método científico, tornou essas culturas inescapáveis à condição de somente objetos de pesquisa e tão somente isso. Abdicando a possibilidade de participação enquanto sujeitos, autores e realizadores. Essa incompreensão é muito bem colocada pelas palavras de Hampaté Bâ em seu texto intitulado "A tradição Viva":

\footnotetext{
Quando falamos de tradição à história Africana, referimo-nos à tradição oral, e nenhuma tentativa de penetrar a história e o espírito dos povos africanos terá validade ao menos que se apoie nessa herança de conhecimentos de toda espécie, pacientemente transmitidos de boca a ouvido..." "...entre as tradições modernas, onde a escrita tem precedência sobre a oralidade, onde o livro constitui o principal veículo da herança cultural, durante muito julgouse que os povos sem escrita eram povos sem cultura [...] (BÂ, 1992, p. 64, apud RAMOS, 2016).
}

Fazemos uma apresentação crítica do problema da produção de conhecimento em ciências humanas e sociais baseado na herança do racionalismo cientificista e tecnicista, para propor uma Produção Partilhada do Conhecimento, que visa ressignificar o lugar das partes envolvidas em pesquisas científicas, retirando do lugar sujeitos-objetos e recolocando enquanto sujeitos-autores inseridos ativamente na pesquisa. 


\section{METODOLOGIA}

Baseado na premissa apresentada acima, abordamos a vivência na comunidade indígena de Sangradouro - MT, com a participação de indígenas Xavantes, Bororos e pesquisadores da USP, onde foram criados quatro vídeos com temáticas sugeridas pelos próprios indígenas, ou seja, a partir da linguagem audiovisual, partilhou-se conhecimentos, saberes e experiências sem estabelecer qualquer relação hierárquica entre comunidade e pesquisadores.

Todos os dias antes das aulas fazíamos uma roda onde cantávamos e dançávamos ao som potente das vozes Xavantes, algo que durava por volta de 10 a 15 minutos, o suficiente para entrarmos numa atmosfera afetiva, coletiva e uma força emocional que era transformada em potência produtiva ao longo do dia, porém com uma sensibilidade e percepção do outro mais aguçada.

Por meio da aproximação, nas oficinas mediada pela linguagem audiovisual, dividimo-nos em 4 grupos e cada qual desenvolveu um roteiro para gravarmos um minidocumentário em cada grupo. Com a duração de 5 minutos cada vídeo, abordamos problemas na Aldeia Xavante de Sangradouro - MT, como o excesso de lixo e a influência da cultura do Warazu (branco) que, de certo modo, contribuem para a não participação em tradições como a Corrida do Buriti. ${ }^{2}$

Buscamos contemplar uma possível intersecção entre conceito e experiência estética, ferramentas digitais e produção de conhecimento, remontando alguns aspectos do antigo debate dicotômico da relação arte/ciência, ciências duras $x$ ciências do espírito, como mencionado em Michel Maffesoli (1998):

[...] era certamente necessário fazer da arte e da ciência "objetos" bem separados: aquela para os sentimentos, esta para a razão, e isso em todos os domínios. As ciências "duras" haviam mostrado o caminho, as ciências humanas deviam segui-lo. Raros foram aqueles que tentaram transgredir tal fronteira; quando o faziam, os riscos e perigos corriam por sua própria conta. (MAFFESOLI, 1998, p. 43).

Porém, pensamos aqui na hipermídia como linguagem que possibilita uma relação heterárquica, ou seja, sem uma hierarquia estabelecida entre Universidade e Comunidade, relação fundamental para condições metodológicas na produção dialógica de conhecimento. As temáticas eram definidas ao longo do dia, em movimento orgânico, onde o acaso era bem-vindo para direcionar nossas discussões conforme precisamos, pois quando planejamos um roteiro de aula, fomos surpreendidos com as intervenções dos "alunos", qualquer ideia perto de uma trilha de aprendizagem era transformada rapidamente em vivências e rodas de conversa de forma espontânea.

Ao final da tarde, os grupos saíam para gravar em busca de contemplar as histórias que surgiam nas rodas de conversa. Quando estávamos na mata ficavam o tempo todo nos protegendo, ensinando os caminhos, alertando possíveis perigos, eram grandes mestres e conhecedores locais.

O senso de comunidade, junto à valorização das subjacências cotidianas é o que nos conduziu no processo para uma produção partilhada do conhecimento, lugar em que o conhecimento científico e o saber de qualquer outro contexto não são

2 Link para acesso aos filmes: TSÕRÉHIPÃRI RE ROMHÖIMANA. Problemas na Aldeia Xavante Sangradouro. Sangradouro, MT: Curso de Formação de Cineastas Indígenas, 2011. 1 vídeo (6:56 min). Disponível em: https://www.youtube.com/watch?v=u 3RdaOw3QA. Acesso em: 15 out. 2021. 
segregados por muros racionalizantes das instituições. Uma ponte entre instâncias comunitárias de saberes plurais e diversos. Em termos metodológicos, é a valorização da experiência estética, da metáfora e do cotidiano reproduzido em ambientes imersivos, em processos de produção de imagens e sons, possibilitados por meio da linguagem hipermídia e audiovisual, que reaproxima o senso comum do saber especializado no processo de produção de conhecimento científico.

\section{RESULTADOS E DISCUSSÃO}

O início das aulas era tocado por essa energia que nos motivava a doar aquilo que tínhamos de melhor com as pessoas que ali nos acolhiam. Assim se seguiu por uma semana entre discussões sobre roteiro, cultura Xavante, Bororo, operação de som, câmera, companheirismo, conflitos políticos na aldeia, muitas vezes com fome, calor e cansaço. Durante o período de duas semanas foram promovidas oficinas diárias entre indígenas Xavantes e Bororos discutindo questões relacionadas ao audiovisual junto aos problemas da aldeia de Sangradouro.

Por meio da aproximação heterárquica mediada pela linguagem audiovisual, dividimo-nos em 4 grupos e cada qual desenvolveu um roteiro para gravarmos um mini-documentário em cada grupo. Tsõréhipãri re Romhöimana, foi o nome dado aos curtas, que significa Índio Misturado, em celebração à presença das diferentes etnias que ali dividiam o mesmo objetivo em comum. Importante lembrar que Xavantes e Bororos são historicamente inimigos territoriais. Com a duração de 5 minutos cada vídeo, abordamos problemas na Aldeia Xavante de Sangradouro - MT, como o excesso de lixo e a influência da cultura do Warazu (branco) que, de certo modo, contribuem para a não participação em tradições como a Corrida do Buriti.

Figura 1 - Ritual de canto Xavante em círculo ao início de todas as atividades.

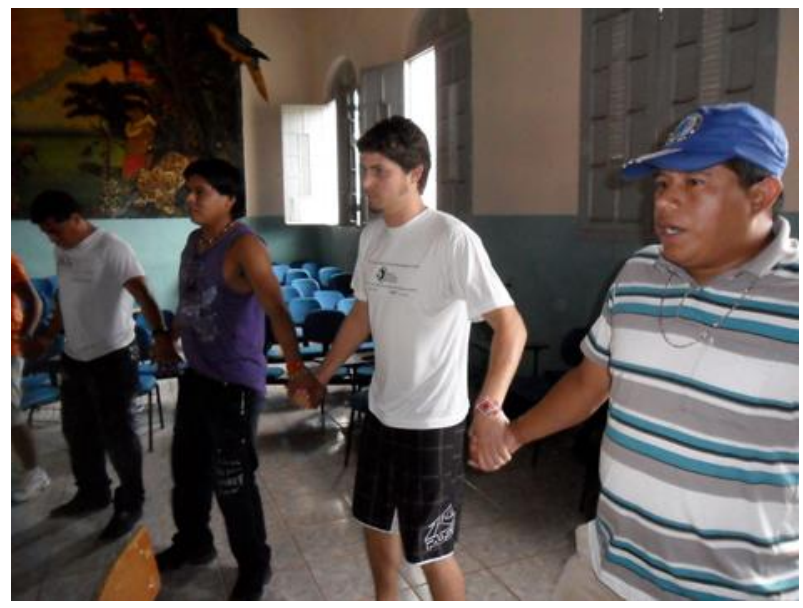

Fonte: Fotografia de Divino Tsereptse Xavante.

Pensamos aqui na hipermídia como linguagem que possibilita uma relação dialógica entre as duas esferas e que proporcione condições metodológicas para uma produção de conhecimento dialógica. Martín-Barbero (2014) coloca em foco o problema do pseudorreconhecimento das narrativas de culturas tradicionais que vivem a oralidade pelas instituições da política oficial, citando uma luta particular com o Ministério da Cultura da Colômbia, que havia criado um prêmio anual de narrativas nacionais voltado à valorização dos mitos indígenas. O problema é que as histórias 
só poderiam concorrer ao prêmio se fossem transcritas para o papel, ou seja, havia a incompreensão da imaterialidade do conhecimento oral e assim 0 valor expressividade da voz como forma de transmissão de saber se fragilizam. $O$ autor ainda indaga:

"Por que as histórias indígenas só podem chegar a uma nação, cujas maiorias continuam vivendo cotidianamente entre cultura oral e a audiovisual, transmutadas em escrita, quando poderiam e deveriam chegar na própria voz - gravada - dos indígenas para que as crianças de todas as escolas do país tivessem a experiência sonora dos outros idiomas que fazem a riqueza do país multiétnico e multicultural que prega a nova Constituição de 1991?" (MARTINBARBERO, 2014, p. 98 Apud, RAMOS, 2016).

Figura 2 - Grupos de trabalho em captação de Imagem na Aldeia.

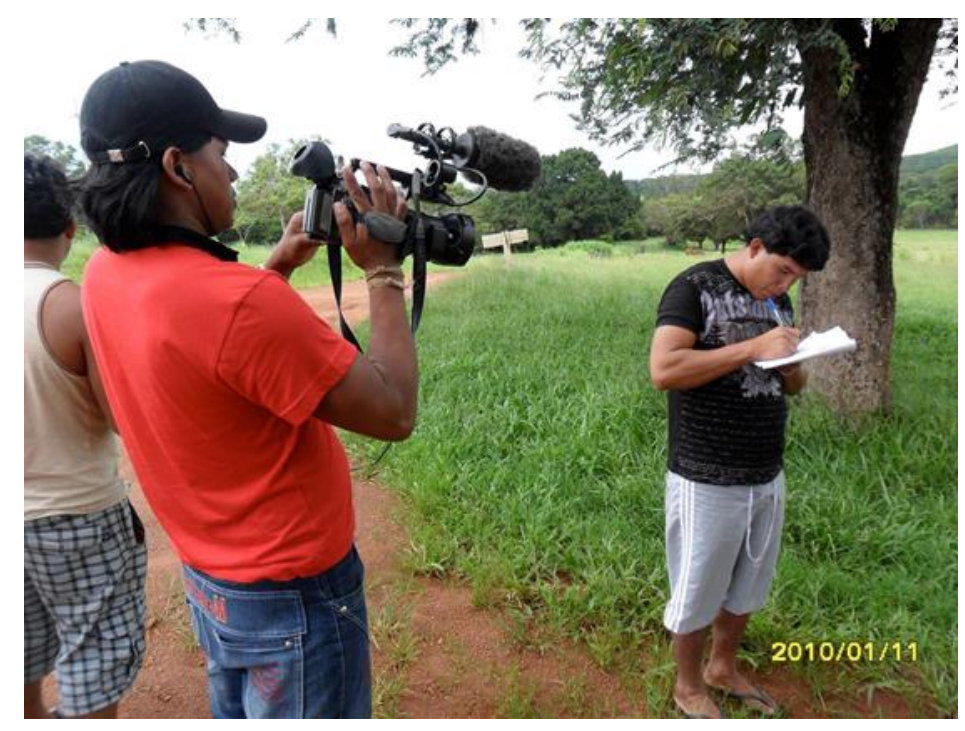

Foto: Jonathas Beck Ramos.

Em seu texto, "Historia, Memoria y Olvido en Los Andes Quechuas" o antropólogo peruano Rodrigo Montoya narra as táticas de transmissão de conhecimento que os povos quechuas praticam para que sua memória não seja perdida e também para engendrar a transmissão de saberes. Dentre elas o autor cita a dança, o teatro, a oralidade e faz uma afirmativa que nos orienta para aquilo que propomos em Produção Partilhada do Conhecimento, ou seja, a valorização das experiências estéticas na vida cotidiana como prática de comunhão de sabedoria.

"Identificar la sabedoría exclusivamente con un alfabeto para escribir y ler una lengua ha sido, sin duda, uno de los errores de más lamentables consecuencias para el desarrollo del conocimiento de la humanidade." (MONTOYA ROJAS, 2014, p.157). 
Figura 3 - Aldeia Xavante de Sangradouro - MT.

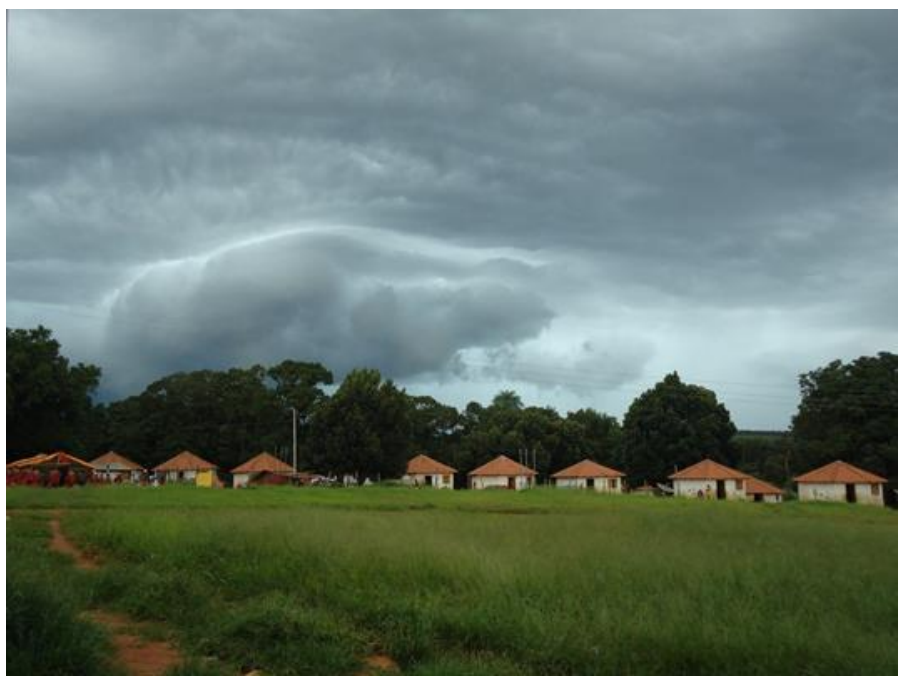

Fonte: Fotografia Rodrigo Pannacci.

Nesta proposta de produção de conhecimento, a partir de linguagens que permitam a valorização de saberes diversos, propomos também a possibilidade de uma abertura para que comunidades diversas possam participar do processo de produção de conhecimento científico, pois se há uma relação outra possível entre saberes díspares de culturas diferentes, podemos neste sentido repensar o lugar do sujeito e objeto nas ciências sociais e humanas. Buscamos esta via associando a experiência estética hipermidiática ao conceito de Produção Partilhada de Conhecimento. Compreendemos junto ao pensamento filosófico de Gadamer, que não podemos considerar apenas o pensamento disciplinar e formal como exclusivo e representativo na constituição da cultura humana:

O que está em questão é muito mais o fato de um modo de pensamento que é distintivo da investigação científica não ser o único e não poder ser o modo de pensamento predominante na administração espiritual da humanidade. Sem dúvida alguma, os gregos também eram uma nação de artesãos de primeiro nível, grandes em inventar, grandes em projetar e grandes em levar a termo. Na terminologia grega, não podemos nem mesmo exprimir a diferença entre o assim chamado artesão e o assim chamado artista livre. Quer denominemos Arquimedes um pesquisador genial, quer um artesão grandioso, trata-se nos dois casos do gênio da techne. (GADAMER, 1991, p. 86) 
Figura 4 - Dayane Boro em oficina de captação de imagem.

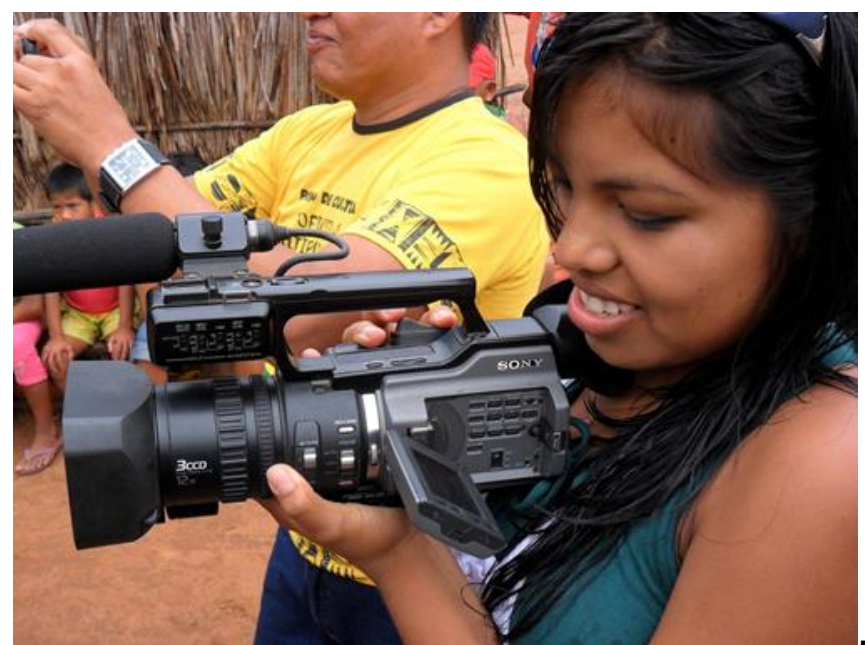

Fonte: Foto de Jonathas Beck Ramos.

Figura 5 - Natal Xavante em oficina de captação de imagem.

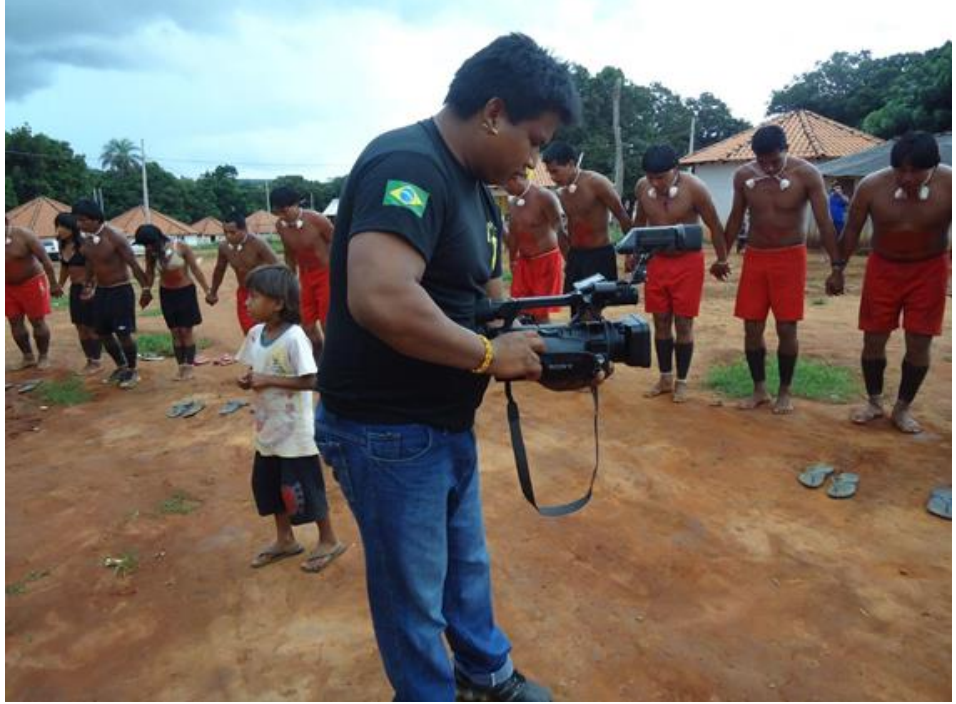

Fonte: Foto de Jonathas Beck Ramos 
Figura 6 - Menino Xavante em oficina de Captação de Imagem.

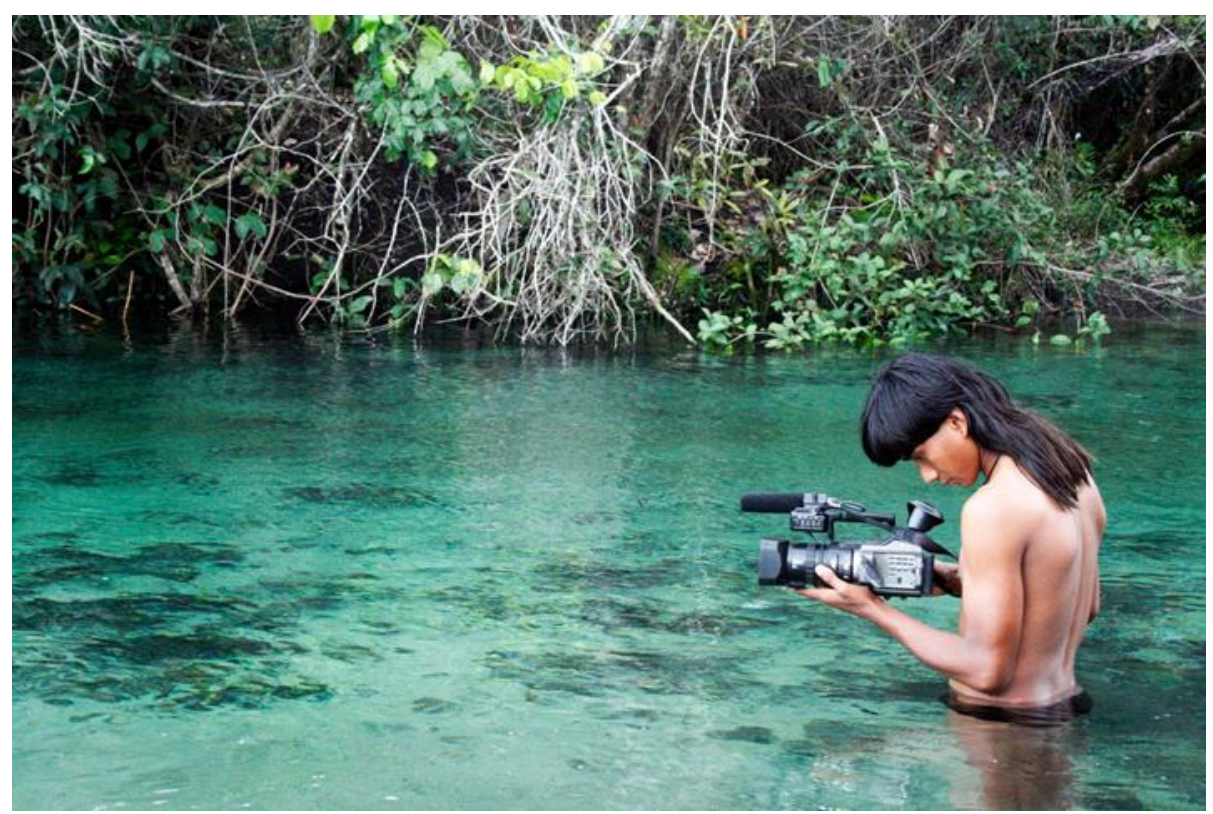

Fonte: Foto de Jonathas Beck Ramos.

\section{CONSIDERAÇÕES FINAIS}

O caminho que apontamos é a possibilidade de pensarmos na linguagem digital hipermidiática, aqui abordada pelo audiovisual, como uma estratégia política de inclusão, de diálogo e, sobretudo, de descolonização do saber calcado em tradições orais, subordinado à cultura racionalizante da escrita. Importante reforçar ainda que as imagens anexadas neste artigo não cumprem com papel apenas ilustrativo do processo descrito, mas são imagens/conceitos e como parte do processo vivenciado e produzido, são inerentes ao conhecimento gerado na dinâmica do encontro entre todas as partes envolvidas na vivência, fruto de uma etnografia da imagem, bem descrito em teorias da antropologia visual de José da Silva Ribeiro (2017). Reconhecemos que a luta por direitos reivindicada por culturas tradicionais orais passa inevitavelmente por conquistas políticas e institucionais no contexto acadêmico, pois é nesses espaços que a cultura da escrita se reafirma permanentemente como status quo de uma noção de cultura baseada na erudição intelectual racionalizante que não permite o reconhecimento e a legitimação institucional de outras formas de saber. Portanto, valorizar o pensamento fora da tradição acadêmica é reconhecer que vivemos em uma América Latina plural e injustiçada pelas instituições.

\section{REFERÊNCIAS}

GADAMER, Hans-Georg. Estética y hermenéutica. Madrid: Technos, 1991.

LAZANEO, Caio. Produção Partilhada do conhecimento: uma experiência com as comunidades indígenas Xavante e Karajá. 2012. Dissertação (Mestrado em Ciência da Comunicação) Universidade de São Paulo, São Paulo, 2012.

MAFFESOLI, Michel. Elogio da razão sensível. Petrópolis, RJ: Vozes, 1998.

MARTíN-BARBERO, Jesús. A Comunicação na Educação. São Paulo: Contexto, 
2014.

MONTOYA ROJAS, Rodrigo. Porvenir de la cultura quechua en Perú: Desde Lima, Villa El Salvador y Puquio. Lima: Fondo Editorial de la Universidad Nacional Mayor de San Marcos, 2010.

RAMOS, Jonathas Beck. Texturas sonoras na Produção Partilhada do

Conhecimento: o áudio na produção de conhecimento em hipermídia. Dissertação (Mestrado), Faculdade de Filosofia, Letras e Ciências Humanas, Universidade de São Paulo, São Paulo, 2016.

RIBEIRO, José da Silva. Pesquisa e Ensino em Antropologia, Antropologia Visual, Antropologia Digital In: LANDA, Mariano Báez; ALVAREZ, Gabriel O (org). Olhar in(com)formado: teorias e práticas na antropologia visual.1 ed. Goiânia: Imprensa Universitária, 2017. p. 102-144. Disponível em:

https://files.cercomp.ufg.br/weby/up/1249/o/EZ8R37 O.PDF. Acesso em: 15 out. 2021. 